\title{
Problems with Double Blind in Medicine
}

\author{
Yngvar Reichelt ${ }^{1}$, Karl L. Reichelt ${ }^{2}$ \\ ${ }^{1}$ Department of Mathematics, University of Oslo, Oslo, Norway \\ ${ }^{2}$ Lab 1, No 1337, Sandvika, Norway and Kleve 4541 m, University of Oslo, Oslo, Norway \\ Email: karlr@ulrik.uio.no
}

Received 5 February 2015; accepted 16 April 2015; published 22 April 2015

Copyright (C) 2015 by authors and Scientific Research Publishing Inc.

This work is licensed under the Creative Commons Attribution International License (CC BY).

http://creativecommons.org/licenses/by/4.0/

(c) (i)

Open Access

\begin{abstract}
Double blind tests of drugs and procedures depend on obtaining two equal and randomly assigned groups to be compared. With a diagnosis based only on symptoms, but with different etiologies, this is not very likely. We here show the probability of obtaining two equal groups with one diagnosis but three etiologies. The mathematical name for such a problem is multivariate hypergeometric distribution. We find that increasing the group size decreases the probability.
\end{abstract}

\section{Keywords}

Diagnosis, Difference, Etiologies, Distribution, Groups

\section{Introduction}

Randomly assigned double blind is the gold standard for therapeutic interventions and procedures in medicine. However, one of the conditions for using this method is that the two compared, randomly assigned groups are equal. Many diagnoses are symptom based, and there is every possibility that a diagnostic entity may have several different causes. This is problematic. We have tried to calculate the chance that a group of patients divided into two groups randomly assigned would be equal, if three different etiologies were subsumed under the same diagnosis.

As an example, decreased serotoninergic activity in the brain can have many different causes. In Figure 1 we can have 1) Decreased release of transmitter into the synaptic cleft; 2) Increased re-uptake from the synaptic cleft; 3) Increased uptake into astroglia from the synaptic cleft; 4) Decreased number of receptors in the post synaptic structure; 5) Decreased receptor sensitivity or inhibition of the same; 6) Inhibition of secondary signals coupled to the receptors such as cyclic AMP and, or cyclic GMP formation; 7) Increased monoamine oxidase activity; 8) Long-term effects of decreased serotonin synthesis [1]. The list can be extended, but it is clear that a decreased serotoninergic state may have several causes. A set of symptoms subsumed under a diagnostic label may well need different interventions. Strongly suspected of having several etiologies are depressions and schizophrenia. This is so because no finding or treatment is found adequate in all individuals in each diagnostic group. 


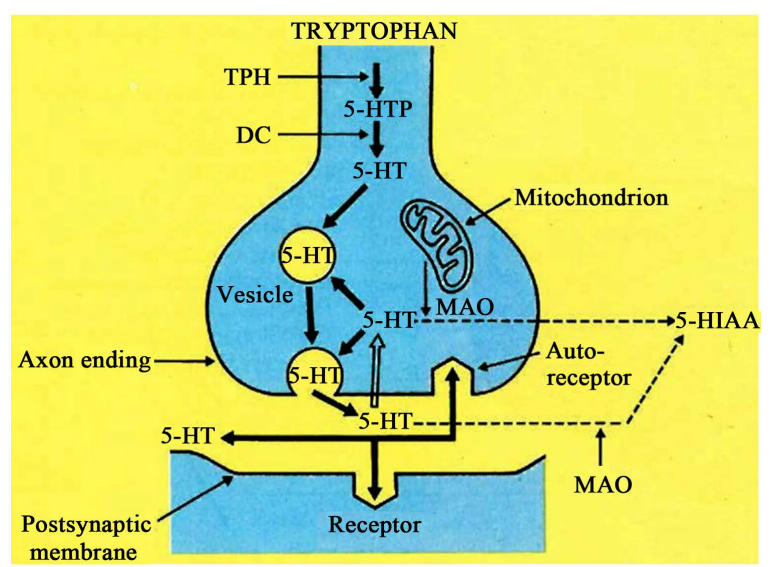

Figure 1. A simplified serotoninergic synapse. 5HT = serotonin; MAO = monoamineoxidase; $\mathrm{TpH}$ is Tryptophan hydroxylase; $5 \mathrm{HTP}=5$ hydroxytryptophan; DC = 5-hydroxytryptophan decarboxylase.

If we randomly pick two and two persons in a group with three different etiologies subsumed under one diagnosis. We remove these two each time to two groups (control and experimental group) - What are the chances of picking two of the same each time? In mathematics this is known as multivariate hypergeometric distribution.

Here we let different colored beads represent the different etiologies.

A: We then have four red, four white and four blue beads with a total of $n=12$.

Then

$$
P(\text { probability })=P(2 \text { red, } 2 \text { white, } 2 \text { blue })=\frac{\left(\begin{array}{l}
4 \\
2
\end{array}\right)\left(\begin{array}{l}
4 \\
2
\end{array}\right)\left(\begin{array}{l}
4 \\
2
\end{array}\right)}{\left(\begin{array}{c}
12 \\
6
\end{array}\right)}=0.2338
$$

where the binomial coefficient is $\left(\begin{array}{l}n \\ k\end{array}\right)=\frac{n !}{(n-k) ! k !}$.

B. If $n=60$ with 20 of each red, white and blue, or

Red $=20$, white $=20$, blue $=20$ then

$P(10 \mathrm{r}, 10 \mathrm{~h}, 10 \mathrm{~b}) \approx 0.0533$

C. If red $=50$, white $=50$ and blue $=50$ and total $n=150$

Then

$P$ (25 red, 25 white and 25 blue $)=0.0218$

C. In general

if $n=$ numbers of marbles; $n / 3=$ numbers of each color (etiologies); $n / 2$ number of marbles to be distributed to each of the random groups

$$
P=\frac{\left(\begin{array}{c}
n / 3 \\
n / 6
\end{array}\right)^{3}}{\left(\begin{array}{c}
n \\
n / 2
\end{array}\right)}
$$

D. Other distributions. In case the frequency of each etiology (here beads) is different.

Red $=12$, white $=6$, blue $=2$ and total $n=20$

$$
P=\frac{\left(\begin{array}{c}
12 \\
6
\end{array}\right)\left(\begin{array}{l}
6 \\
3
\end{array}\right)\left(\begin{array}{l}
2 \\
1
\end{array}\right)}{\left(\begin{array}{l}
20 \\
10
\end{array}\right)} \approx 0.2000
$$


E-increasing the total to $n=60$

Red $=36$, white $=18$, blue $=6$

$$
P=\frac{\left(\begin{array}{c}
36 \\
18
\end{array}\right)\left(\begin{array}{c}
18 \\
6
\end{array}\right)\left(\begin{array}{l}
6 \\
3
\end{array}\right)}{\left(\begin{array}{l}
60 \\
30
\end{array}\right)} \approx 0.0746
$$

\section{Discussion}

Mathematically it is clear that with different etiologies (here beads) subsumed under one diagnosis, then the random distribution into two equal groups is improbable. It is also clear from the calculations done that increasing the numbers, decreases the probability of getting two equal groups with the same distribution.

Based on symptoms alone without a common marker this is hazardous at best. Pneumonia has very many different etiologies, but with microbial diagnosis (markers) this fairly large group can now be divided into etiological sub-groups. Specific treatment is hence more likely to be successful.

\section{Author Contributions}

a) Mathematics written by Yngvar Reichelt;

b) Neurochemical example and final Ms by Karl L. Reichelt.

\section{Funding}

No funding.

\section{Conflict of Interest}

None.

\section{References}

[1] Lehman, J. (1972) Mental and Neuromuscular Symptoms in Tryptophan Deficiency. Acta Psychiatrica Scandinavica, Suppl. 237. 